\title{
Calculated vibration frequencies and IR absorption intensities of [6] cyclacene (zig zag) molecule
}

\author{
Rehab M. Kubba, Huda N. AL-Ani and Muthana Shanshal \\ Department of Chemistry, College of Science, University of Baghdad, Baghdad, Iraq.
}

\begin{abstract}
The (3N-6) vibration frequencies and IR-absorption intensities of [6] Cyclacene (zig zag) molecule were calculated applying Density Functional Theory (DFT) of the type (B3LYP) and a Gaussian basis $(6-311 \mathrm{G}$ ) as well as the semiemperical PM3 method. Comparison of the results showed that similar to the polyaromatic hydrocarbons, the following relations hold: $v_{\text {sym. }} \mathrm{CH}$ str. > $v_{\text {asym. }} \mathrm{CH}$ str. and, $v_{\text {sym }}$. CC str. $>v_{\text {asym. }}$ CC str. i. e., $v_{\text {sym. }}$ CC str. (axial.) $>v_{\text {asym. }}$ CC str. (axial) $>$ $v_{\text {asym. }}$. CC str. (circumferential) where axial are the vertical C-C bonds (annular bonds) in the rings

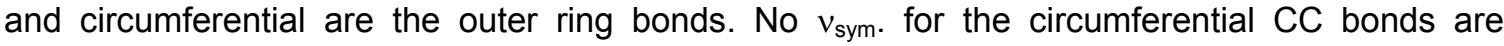
detected. The results include the assignment of all puckering, breathing and clock-anticlockwise bending vibrations. They allow a comparative view of the charge density at the carbon atoms too.
\end{abstract}

\section{INTRODUCTION}

Single wall carbon nanotubes (SWCNTs) are formed from folded sheets of annulated six membered aromatic rings [1]. The hybridization of each carbon atom in the sheet is $\mathrm{sp}^{2}$ [2]. They are of aromatic character, similar to graphite, and posse's conjugated C-C bonds [3]. Their aromaticity is graded according to the space distribution of the atoms, the nature of their molecular orbitals, their symmetry and chirality [4]. Various studies were done for the physical properties of the nanotubes [5-10].

Cyclacenes are monoring segments of carbon nano tubes (CNT), Fig.1. Generally, attempts to synthesize them failed, with the exceptions of few cases [11]. An approach for their chemical stabilization was suggested [12]. It should facilitate their synthesis. For the cyclacene molecules with different ring size, numerous theoretical studies had been published [11]. However, no thorough treatment for their vibration frequencies appeared. Comparison of their vibration frequency values with those of the nanotubes and the planar polyaromatic hydrocarbons should help to understand their general force field.
For this purpose a complete normal coordinate analysis is required. In this work the quantum mechanical Density Functional Theory (DFT) method [13], is applied, in the form of the B3LYP approach [14] and the 6-311G Gaussian bases [15] is utilized. For comparison purposes semiempirical PM3 [16] calculations were done too.

No study could be found in the literature for a complete normal coordinate analysis of the simplest type of CNT i.e. the cyclacene molecule. The following paragraphs describe an attempt for such a study.

Methods of calculation: Both G03 program of Pople et al. [17] and the Molek9000 program of P. Bischof, Heidelberg, [18] were applied.

\section{RESULTS AND DISCUSSION}

To study the vibration motions of such molecules, one has to define its geometric parameters, and has to distinguish between the axial CC (C-Caxial) bonds and circumferential $\mathrm{CC}(\mathrm{C}-\mathrm{Cc})$ bonds. Figure 1 shows the two types of bonds in a zig zag nanoring.

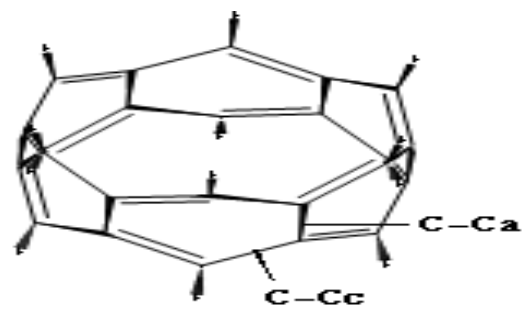

Fig. 1: Structure of [6] Cyclacene (zig zag) molecule, indicating the two types of C-C bonds; C-Caxial (C-Ca) and CCcircumferential (C-Cc). 
Obviously, the vibration of a nanotube as well as cyclacenes, causes a change in its geometry Fig. 2.
Structure deformation is expected to change their thermal and electronic properties [19] too.
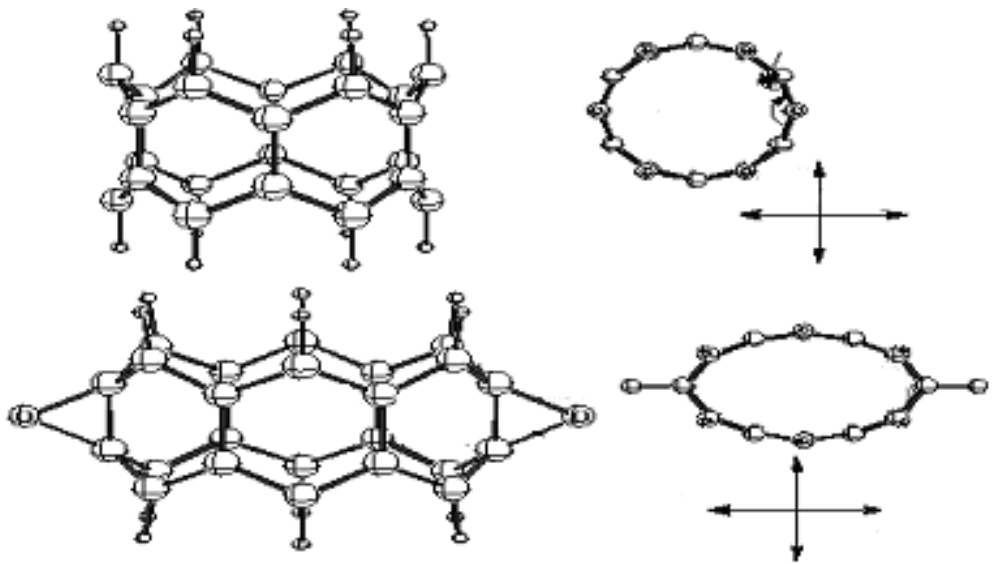

Fig. 2: Structure deformation of a [6] Cyclacene (zig zag) molecule as caused by its vibration motion.

Basic vibrations of CNTs were measured and assigned as breathing, puckering and clock-anticlockwise deformation modes [20]. The frequencies of their active vibrations range between (873-1557 $\mathrm{cm}^{-1}$ ) [21]. They are considered as finger print vibrations for the carbon nanotubes (CNTs) [22]. Measurements were done at $6^{\circ} \mathrm{K}$ to study the impact of the puckering distortion on the electronic properties of CNTs [23-24].

The [6] cyclacene (zig zag) molecule is composed of annulated six membered aromatic rings. It's (DFT) or semiempirical (PM3) calculated equilibrium geometry shows a $D_{6 h}$ symmetry, (Fig. 3).
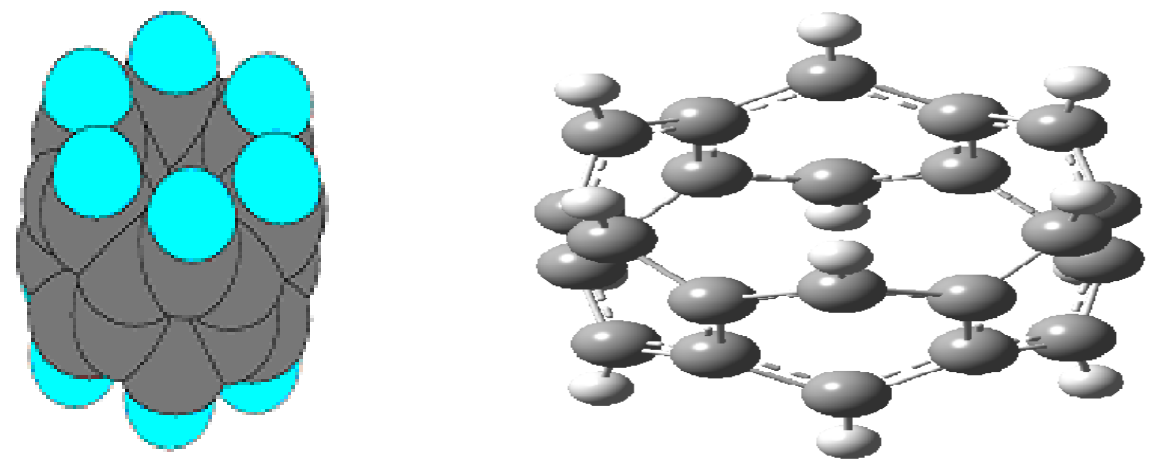

Fig. 3: Equilibrium geometry for [6] Cyclacene (zig zag) molecule.

Due to its symmetry $\left(D_{6 h}\right)$ it undergoes the following symmetry operations $\left(\mathrm{E}, 2 \mathrm{C}_{6}, 2 \mathrm{C}_{3}, \mathrm{C}_{2}, 3 \mathrm{C}_{2}{ }_{2}, 3 \mathrm{C}^{\prime \prime}{ }_{2}\right.$, $\mathrm{i}, 2 \mathrm{~S}_{3}, 2 \mathrm{~S}_{6}, \sigma_{\mathrm{h}}, 3 \sigma_{\mathrm{d}}, 3 \sigma_{\mathrm{v}}$ ). Fig. 4 shows a section of 6-cyclacene molecule, and Table 1 shows its calculated geometric parameters. 
Table (1): Calculated geometry for [6] (Cyclacene (zig-zag)) molecule.

*: circumferential bond.

$* *$ : axial bond.

\begin{tabular}{|l|c|c|}
\hline $\begin{array}{c}\text { Bond length }(\AA) \\
\text { and } \\
\begin{array}{c}\text { Bond angles } \\
\text { (deg.) }\end{array}\end{array}$ & (PM3) & $\begin{array}{c}\text { (DFT) B3LYPI } \\
6-311 G\end{array}$ \\
\hline $\mathrm{C}_{1} \mathrm{C}_{2}{ }^{*}$ & 1.441 & 1.413 \\
\hline $\mathrm{C}_{1} \mathrm{C}_{3}{ }^{*}$ & 1.386 & 1.413 \\
\hline $\mathrm{C}_{2} \mathrm{C}_{4}{ }^{* *}$ & 1.446 & 1.446 \\
\hline $\mathrm{C}_{3} \mathrm{C}_{5}{ }^{* *}$ & 1.446 & 1.446 \\
\hline $\mathrm{C}_{4} \mathrm{C}_{6}{ }^{*}$ & 1.441 & 1.413 \\
\hline $\mathrm{C}_{5} \mathrm{C}_{6}{ }^{*}$ & 1.386 & 1.413 \\
\hline $\mathrm{C}_{1} \mathrm{H}_{7}$ & 1.095 & 1.095 \\
\hline $\mathrm{C}_{6} \mathrm{H}_{8}$ & 1.095 & 1.095 \\
\hline$<\mathrm{C}_{2} \mathrm{C}_{1} \mathrm{C}_{3}$ & 113.829 & 113.855 \\
\hline$<\mathrm{C}_{2} \mathrm{C}_{1} \mathrm{H}_{7}$ & 120.300 & 121.281 \\
\hline$<\mathrm{C}_{1} \mathrm{C}_{2} \mathrm{C}_{4}$ & 119.566 & 118.934 \\
\hline$<\mathrm{C}_{1} \mathrm{C}_{2} \mathrm{C}_{10}$ & 116.812 & 116.844 \\
\hline$<\mathrm{C}_{1} \mathrm{C}_{3} \mathrm{C}_{5}$ & 119.573 & 118.934 \\
\hline$<\mathrm{C}_{1} \mathrm{C}_{3} \mathrm{C}_{9}$ & 116.821 & 116.844 \\
\hline
\end{tabular}

Vibration frequencies assignment of [6] Cyclacene (zig zag) molecule.

The [6] Cyclacene (zig-zag) molecule posses 102 fundamental vibrations. Inspection of its irreducible representation, as defined by the symmetry character table, results in the following modes of vibration;

$\Gamma_{\text {vibration }}=\Gamma_{\text {total }}-\left(\Gamma_{\text {rotation }}+\Gamma_{\text {translation }}\right)=3 \mathrm{~N}-6=108-$ $6=102=6 A_{1 g}+2 A_{2 g}+4 B_{1 u}+5 B_{2 u}+8 E_{1 u}+9 E_{2 g}+$ $3 A_{1 u}+5 A_{2 u}+5 B_{1 g}+4 B_{2 g}+8 E_{1 g}+9 E_{2 u}$

Relative to the $\sigma_{\mathrm{h}}$ reflection the vibration modes are classified as symmetric and antisymmetri modes;

a- symmetric modes with respect to $\sigma_{h}\left(+\sigma_{h}\right)$. $\Gamma_{+6 h}=6 A_{1 g}+2 A_{2 g}+5 B_{1 u}+4 B_{2 u}+8 E_{1 u}+9 E_{2 g}$ (Inplane $\sigma_{h}$ modes of vibrations)

b- antisymmetric modes with respect to $\sigma_{h}\left(-\sigma_{h}\right)$.. $\Gamma_{-б \mathrm{~h}}=3 \mathrm{~A}_{1 \mathrm{u}}+5 \mathrm{~A}_{2 \mathrm{u}}+4 \mathrm{~B}_{1 \mathrm{~g}}+5 \mathrm{~B}_{2 \mathrm{~g}}+8 \mathrm{E}_{1 \mathrm{~g}}+9 \mathrm{E}_{2 \mathrm{u}}$ (Out of plane $\sigma_{\mathrm{h}}$ modes of vibrations)

1- Frequencies of the symmetric modes of vibration $\left(+\sigma_{h}\right)$

These are 53 modes of vibration in number, of which 24 are Raman active $\left(6 \mathrm{~A}_{1 \mathrm{~g}}\right.$ and $\left.9 \mathrm{E}_{2 \mathrm{~g}}\right)$, and $18 \mathrm{IR}$ active $\left(9 \mathrm{E}_{1 \mathrm{u}}\right)$. Their assignments are as follows:

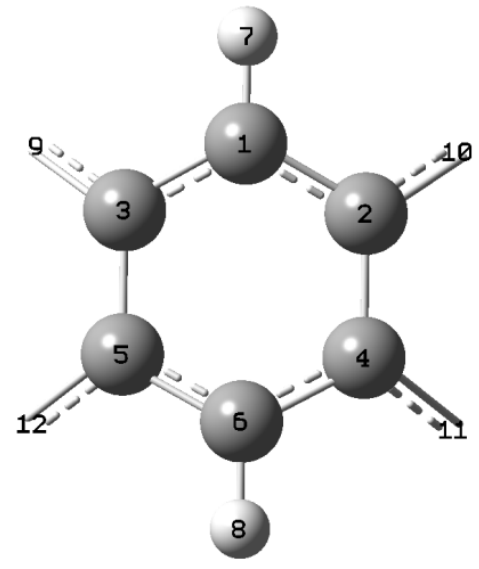

Fig. 4: Repetitive section of the bonds and angles of [6] Cyclacene (zig zag) molecule.

$\mathrm{CH}$ stretching vibrations

These are 12 in number, the displacement vectors of which are located at the $\mathrm{H}$ atoms. Their frequency values range from (3071-3073 $\left.\mathrm{cm}^{-1}\right)$ (PM3) or (3055$3067 \mathrm{~cm}^{-1}$ ) (DFT). Inspecting the values in Table 2 one detects the following correlations:

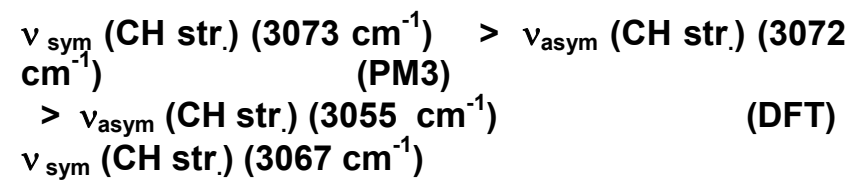

(DFT)

\section{Ring (CC stretching) vibrations}

These are 16 in number. Their displacement vectors are mainly located at the carbon atoms of the related bonds. Their calculated vibration frequencies range from (1479-1717 $\mathrm{cm}^{-1}$ ) (PM3) or $\left(1382-1565 \mathrm{~cm}^{-1}\right)$ (DFT). They show the following relations;

$v_{\text {sym. }}\left(\right.$ CC str.) $\left(1644 \mathrm{~cm}^{-1}\right)$ (axial.) $>v_{\text {asym. }}($ CC str.) $\left(1583 \mathrm{~cm}^{-1}\right)$ (axial.) (PM3) $v_{\text {sym. }}\left(\right.$ CC str.) $\left(1644 \mathrm{~cm}^{-1}\right)$ (axial.) $>v_{\text {asym. }}$ (CC str.) (1600 $\left.\mathrm{cm}^{-1}\right)$ (circum.) (PM3)

$v_{\text {sym. }}\left(\right.$ CC str.) $\left(1531 \mathrm{~cm}^{-1}\right)$ (axial.) > $v_{\text {asym. }}($ CC str.) $\left(1457 \mathrm{~cm}^{-1}\right)$ (axial.) (DFT)

$$
\left(A_{1 g}\right)
$$




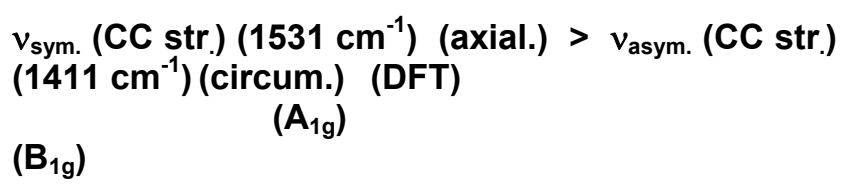

Ring (CCC stretching vibrations)

Unlike the C-C vibration modes, these are not located at definite $C$ atoms as could be seen from the atomic displacement vectors. Their frequencies are lower than those of the $\mathrm{C}-\mathrm{C}$ vibration modes. According to the calculating results (Table 2), they range from $\left(1248-1479 \mathrm{~cm}^{-1}\right)(\mathrm{PM} 3)$ or $\left(1300-1347 \mathrm{~cm}^{-1}\right)(\mathrm{DFT})$.

\section{Bending (CCC) vibrations ( $\delta \mathrm{CCC}$ )}

Of smaller values are the deformation ( $\delta C C C)$ vibrations. According to their assignment, they fall in the range $\left(453-1196 \mathrm{~cm}^{-1}\right)(\mathrm{PM} 3)$ or $\left(431-1228 \mathrm{~cm}^{-1}\right)$ (DFT). These modes include the expected clock and anticlockwise vibration motions. Their symmetric modes are of higher frequencies than the asymmetric, e. g. in the following scheme;

$v_{\text {sym. }}\left(\right.$ ( CCC) $\left(890 \mathrm{~cm}^{-1}\right)>v_{\text {asym. }}($ ( CCC) $)\left(453 \mathrm{~cm}^{-1}\right)$ (PM3)

and,

$v_{\text {sym. }}(\delta C C C)\left(775 \mathrm{~cm}^{-1}\right)>v_{\text {asym. }}\left(\right.$ (CCC) $\left(431 \mathrm{~cm}^{-1}\right)$ (DFT)

\section{Bending $\mathrm{CH}$ vibrations $(\delta \mathrm{CH})$}

There are $12 \mathrm{\delta CH}$ vibration modes, as few as the C$\mathrm{H}$ bonds. Their displacement vectors are mainly located at the corresponding $\mathrm{H}$ atoms. Their calculated frequency values range from (1119-1384 $\left.\mathrm{cm}^{-1}\right)(\mathrm{PM} 3)$ or $\left(1107-1288 \mathrm{~cm}^{-1}\right)$ (DFT) Table 2.

2- Frequencies of the asymmetric modes of vibration $\left(-\sigma_{h}\right)$.

These are 49 modes 16 of them are Raman active $\left(8 \mathrm{E}_{1 \mathrm{~g}}\right)$ and $5 \mathrm{IR}$ active $\left(\mathrm{A}_{2 \mathrm{u}}\right)$, their assignment is discussed in the following paragraph.

The $(\gamma \mathrm{CH})$ out of plane vibration frequencies range from $\left(782-950 \mathrm{~cm}^{-1}\right)(\mathrm{PM} 3)$ or $\left(817-956 \mathrm{~cm}^{-1}\right)$ (DFT), see Table 2. The following relations hold too;

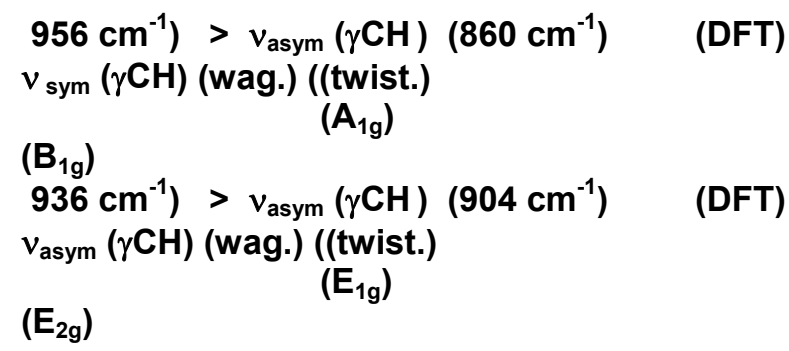

The ring out of plane vibrations $(\gamma \mathrm{CCC}), 23$ modes, the calculated frequencies of which range from (147$\left.827 \mathrm{~cm}^{-1}\right)$ (PM3) or (133-768 $\mathrm{cm}^{-1}$ ) (DFT). The modes include puckering deformations, as well as breathing vibrations of the whole ring. The relation of the sym. to the asym. modes are viewed in the following scheme;

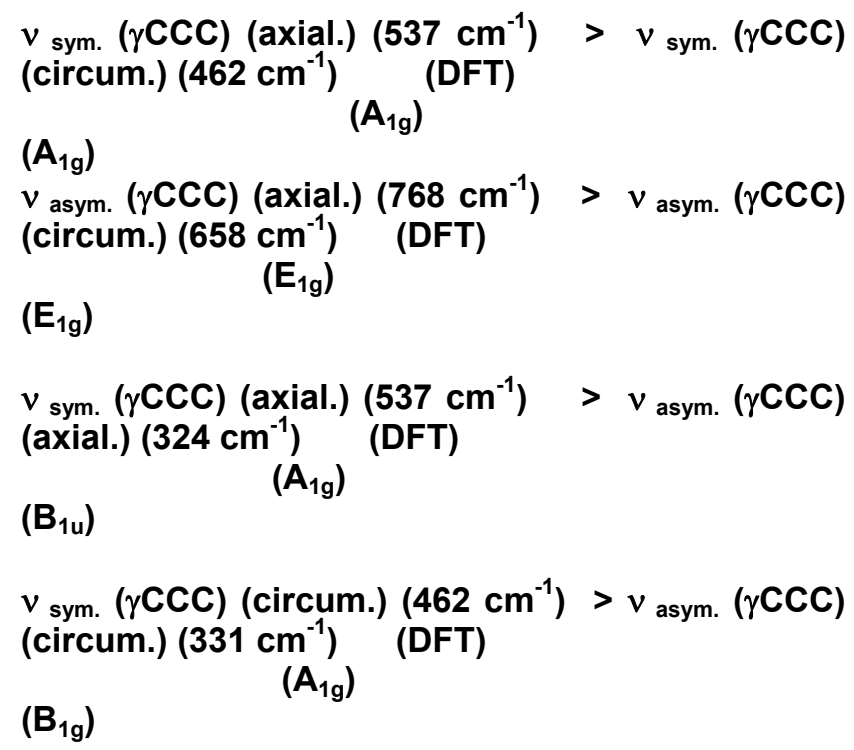

Table 2 includes the calculated frequencies and IR absorption intensities of the vibration modes. Commonly known, the vibrations with (intensity $=0.0$ ) are forbidden, those with (intensity $\neq 0.0$ ) are allowed. The vibration modes in the table are classified according to the Herzberg scheme [25].

Figure 5 shows the vibration pictures for some modes of the [6] Cyclacene molecule, as calculated applying the DFT method. 
Am. J. Sci. Ind. Res., 2011, 2(4): 642-651

Table (2): Calculated vibration frequencies and IR absorption intensities for [6] Cyclacene (zig zag) molecule.

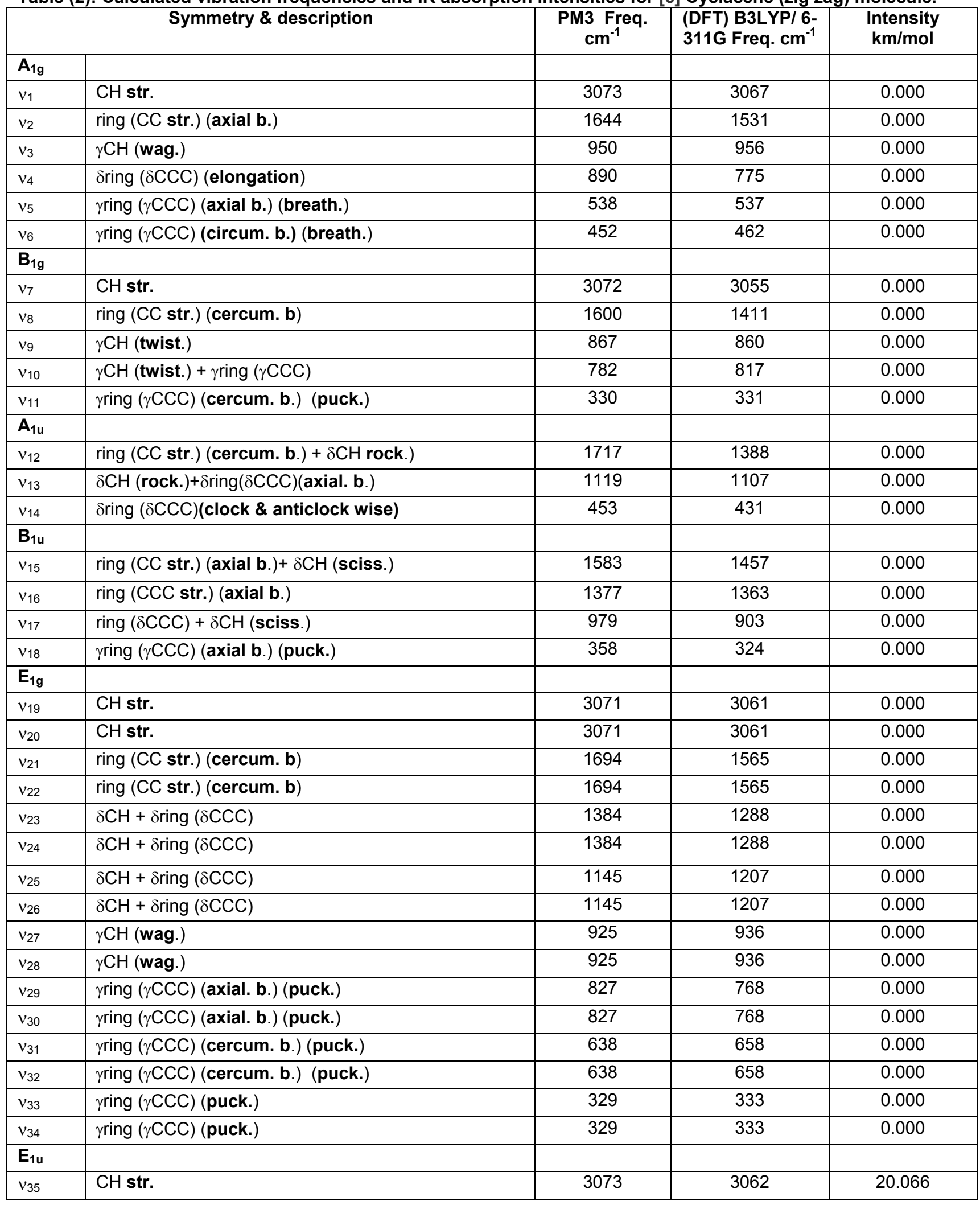


Am. J. Sci. Ind. Res., 2011, 2(4): 642-651

\begin{tabular}{|c|c|c|c|c|}
\hline$v_{36}$ & $\mathrm{CH}$ str. & 3073 & 3062 & 20.066 \\
\hline$v_{37}$ & ring (CC str.) (axial b.) $+\delta C H$ (rock.) & 1632 & 1516 & 3.559 \\
\hline$v_{38}$ & ring (CC str.) (axial b.) $+\delta C H$ (rock.) & 1632 & 1516 & 3.559 \\
\hline$v_{39}$ & ring (CCC str.) (axial b.) & 1479 & 1347 & 32.422 \\
\hline$v_{40}$ & ring (CCC str.) (axial b.) & 1479 & 1347 & 32.422 \\
\hline$v_{41}$ & $\delta \mathrm{CH}$ (rock.) + ring (CCC str.) & 1156 & 1201 & 0.117 \\
\hline$v_{42}$ & $\delta \mathrm{CH}$ (rock.) + ring (CCC str.) & 1156 & 1201 & 0.117 \\
\hline$v_{43}$ & $\gamma \mathrm{CH}$ (twist.) & 927 & 910 & 557.652 \\
\hline$v_{44}$ & $\gamma \mathrm{CH}$ (twist.) & 927 & 910 & 557.652 \\
\hline$v_{45}$ & fring $(\delta C C C)$ (axial b.) (elongation) & 894 & 762 & 5.054 \\
\hline$v_{46}$ & fring $(\delta C C C)$ (axial b.) (elongation) & 894 & 762 & 5.054 \\
\hline$v_{47}$ & fring $(\delta C C C)$ (cercum. b.) & 594 & 585 & 19.554 \\
\hline$v_{48}$ & Sring $(\delta C C C)$ (cercum. b.) & 594 & 585 & 19.554 \\
\hline$v_{49}$ & $\gamma$ ring $(\gamma C C C)$ (cercum. b.) (puck.) & 443 & 403 & 8.433 \\
\hline$v_{50}$ & $\gamma$ ring $(\gamma \mathrm{CCC})$ (cercum. b.) (puck.) & 443 & 403 & 8.433 \\
\hline \multicolumn{5}{|c|}{ 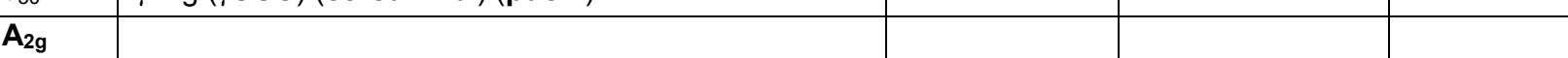 } \\
\hline$v_{51}$ & $\begin{array}{l}\text { ring (CC str.) (circum. b., clock \& anti clockwise) + } \\
\delta \mathrm{CH}\end{array}$ & 1248 & 1504 & 0.000 \\
\hline$v_{52}$ & $\delta \mathrm{CH}$ (rock.) (clock \& anticlockwise) & 1090 & 1227 & 0.000 \\
\hline \multicolumn{5}{|l|}{$A_{2 u}$} \\
\hline$v_{53}$ & $\mathrm{CH}$ str. & 3072 & 3064 & 58.588 \\
\hline$v_{54}$ & ring (CCC str.) (cercum. b.) $+\delta \mathrm{CH}$ & 1404 & 1300 & 90.410 \\
\hline$v_{55}$ & $\gamma \mathrm{CH}$ (wag.) & 928 & 922 & 378.955 \\
\hline$v_{56}$ & rring $(\gamma C C C)$ (axial. b.) (puck.) & 809 & 735 & 131.157 \\
\hline$v_{57}$ & rring $(\gamma \mathrm{CCC})$ (cercum. b.) (puck.) & 418 & 382 & 76.438 \\
\hline \multicolumn{5}{|l|}{$B_{2 g}$} \\
\hline$v_{58}$ & ring (CC str.) (cercum. b) & 1556 & 1432 & 0.000 \\
\hline$v_{59}$ & $\delta \mathrm{CH}$ (sciss.) + ring $(\delta \mathrm{CCC})$ & 1207 & 1274 & 0.000 \\
\hline$v_{60}$ & pring $(\gamma C C C)$ (axial. b.) (puck.) & 678 & 665 & 0.000 \\
\hline$v_{61}$ & ring $(\delta C C C)$ (cercum. b.) & 475 & 466 & 0.000 \\
\hline \multicolumn{5}{|l|}{$B_{2 u}$} \\
\hline$v_{62}$ & $\mathrm{CH}$ str. & 3073 & 3056 & 0.000 \\
\hline$v_{63}$ & ring (CCC str.) $+\delta \mathrm{CH}$ (sciss.) & 1573 & 1320 & 0.000 \\
\hline$v_{64}$ & $\gamma \mathrm{CH}$ (twist.) & 832 & 876 & 0.000 \\
\hline$v_{65}$ & ring $(\delta \mathrm{CCC})+\delta \mathrm{CH}$ (sciss.) & 598 & 599 & 0.000 \\
\hline$v_{66}$ & pring $(\gamma C C C)$ (axial b.) (puck.) & 281 & 255 & 0.000 \\
\hline \multicolumn{5}{|l|}{$E_{2 g}$} \\
\hline$v_{67}$ & $\mathrm{CH}$ str. & 3073 & 3058 & 0.000 \\
\hline$v_{68}$ & $\mathrm{CH}$ str. & 3073 & 3058 & 0.000 \\
\hline$v_{69}$ & ring (CC str.)(axial b.) $+\delta \mathrm{CH}$ (sciss.) & 1608 & 1497 & 0.000 \\
\hline$v_{70}$ & ring (CC str.)(axial b.) $+\delta \mathrm{CH}$ (sciss.) & 1608 & 1497 & 0.000 \\
\hline
\end{tabular}


Am. J. Sci. Ind. Res., 2011, 2(4): 642-651

\begin{tabular}{|c|c|c|c|c|}
\hline$v_{71}$ & ring (CC str.) (axial b. + cercum. b.) & 1576 & 1382 & 0.000 \\
\hline$v_{72}$ & ring (CC str.) (axial b.+ cercum. b.) & 1576 & 1382 & 0.000 \\
\hline$v_{73}$ & $\delta \mathrm{CH}+(\delta \mathrm{CCC})$ & 1280 & 1271 & 0.000 \\
\hline$v_{74}$ & $\delta \mathrm{CH}+(\delta \mathrm{CCC})$ & 1280 & 1271 & 0.000 \\
\hline$v_{75}$ & $\gamma \mathrm{CH}$ (twist.) & 961 & 904 & 0.000 \\
\hline$v_{76}$ & $\gamma \mathrm{CH}$ (twist.) & 961 & 904 & 0.000 \\
\hline$v_{77}$ & $\gamma \mathrm{CH}$ (twist. $)+\gamma$ ring $(\gamma \mathrm{CCC})$ & 864 & 845 & 0.000 \\
\hline$v_{78}$ & $\gamma \mathrm{CH}$ (twist. $)+\gamma$ ring $(\gamma \mathrm{CCC})$ & 864 & 845 & 0.000 \\
\hline$v_{79}$ & dring ( $\delta C C C)$ (circum b.) (elongation) & 627 & 619 & 0.000 \\
\hline$v_{80}$ & Sring ( $\delta \mathrm{CCC})$ (circum b.) (elongation) & 627 & 619 & 0.000 \\
\hline$v_{81}$ & pring ( $\gamma$ CCC) (cercum. b.) (puck.) & 405 & 387 & 0.000 \\
\hline$v_{82}$ & $\gamma$ ring $(\gamma \mathrm{CCC})$ (cercum. b.) (puck.) & 405 & 387 & 0.000 \\
\hline$v_{83}$ & $\gamma$ ring $(\gamma C C C)$ (axial b.) (puck.) & 147 & 133 & 0.000 \\
\hline$v_{84}$ & pring ( $\gamma$ CCC) (axial b.) (puck.) & 147 & 133 & 0.000 \\
\hline \multicolumn{5}{|l|}{$E_{2 u}$} \\
\hline$v_{85}$ & $\mathrm{CH}$ str. & 3072 & 3056 & 0.000 \\
\hline$v_{86}$ & CH str. & 3072 & 3056 & 0.000 \\
\hline$v_{87}$ & ring (CC str.) (cercum. b) & 1685 & 1547 & 0.000 \\
\hline$v_{88}$ & ring (CC str.) (cercum. b) & 1685 & 1547 & 0.000 \\
\hline$v_{89}$ & ring (CCC str.) $+\delta \mathrm{CH}$ (sciss.) & 1428 & 1308 & 0.000 \\
\hline$v_{90}$ & ring (CCC str.) $+\delta \mathrm{CH}$ (sciss.) & 1428 & 1308 & 0.000 \\
\hline$v_{91}$ & Sring $(\delta C C C)$ (cercum. b) & 1196 & 1228 & 0.000 \\
\hline$v_{92}$ & Sring ( $\delta C C C)$ (cercum. b) & 1196 & 1228 & 0.000 \\
\hline$v_{93}$ & $\gamma \mathrm{CH}$ (twist.) & 885 & 892 & 0.000 \\
\hline$v_{94}$ & $\gamma \mathrm{CH}$ (twist.) & 885 & 892 & 0.000 \\
\hline$v_{95}$ & $\gamma \mathrm{CH}$ (twist. $)+\gamma$ ring $(\gamma \mathrm{CCC})$ & 846 & 839 & 0.000 \\
\hline$v_{96}$ & $\gamma \mathrm{CH}$ (twist. $)+\gamma$ ring $(\gamma \mathrm{CCC})$ & 846 & 839 & 0.000 \\
\hline$v_{97}$ & $\gamma$ ring $(\gamma \mathrm{CCC})$ (axial. b.) (puck.) & 672 & 670 & 0.000 \\
\hline V98 & $\gamma$ ring $(\gamma \mathrm{CCC})$ (axial. b.) (puck.) & 672 & 670 & 0.000 \\
\hline$v_{99}$ & $\gamma$ ring $(\gamma \mathrm{CCC})$ (cercum. b) (puck.) & 430 & 429 & 0.000 \\
\hline$v_{100}$ & $\gamma$ ring $(\gamma \mathrm{CCC})$ (cercum. b) (puck.) & 430 & 429 & 0.000 \\
\hline$v_{101}$ & pring $(\gamma \mathrm{CCC})$ (cercum. b) (puck.) & 181 & 185 & 0.000 \\
\hline$v_{102}$ & $\gamma$ ring $(\gamma \mathrm{CCC})$ (cercum. b) (puck.) & 181 & 185 & 0.000 \\
\hline
\end{tabular}

$\gamma$ : Out of plane of the molecule.

$\delta$ : In- plane of the molecule.

(breath.): ring breathing mode.

(puck.): ring puckering mode.

(rock.): $\mathrm{CH}$ rocking mode.

(sciss.): $\mathrm{CH}$ scissoring mode.

(twist.): $\mathrm{CH}$ twisting mode.

(wag.): $\mathrm{CH}$ wagging mode. 

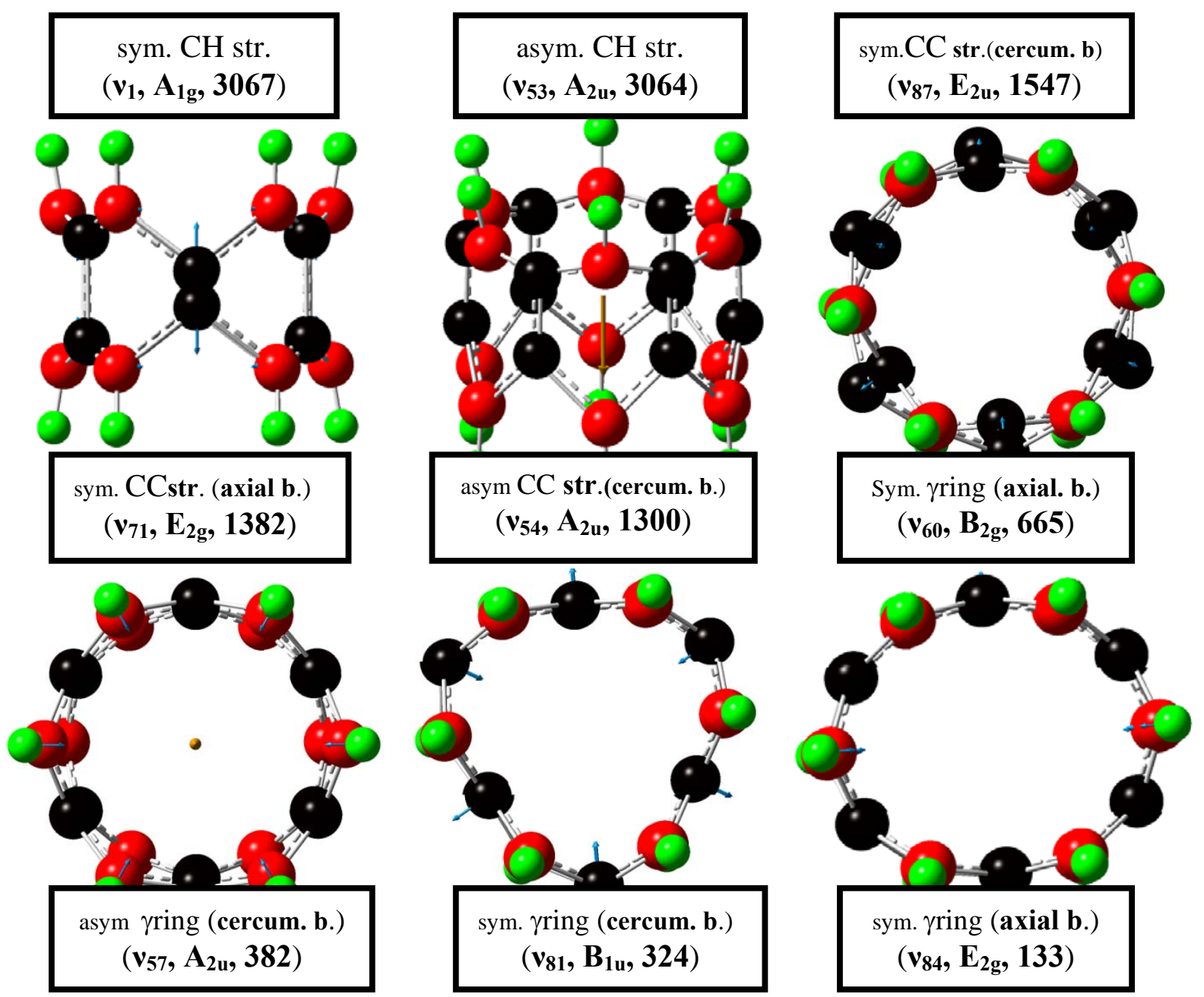

Fig.5. The graphical pictures of some vibration modes for [6] Cyclacene (zig zag) molecule as calculated applying the DFT method.

Compared with the frequencies of anthracene molecule, as calculated applying the same DFT method and gauss basis, the frequency values of [6] cyclacene are lower (table-3). The comparison shows that the force field for cyclacene is weaker than that of the coplanar polyaromatic molecule. It indicates the influence of folding in diminishing the $\mathrm{C}-\mathrm{C}$ bond strength of the aromatic molecule too. 
Am. J. Sci. Ind. Res., 2011, 2(4): 642-651

Table 3: Comparison of the vibration frequencies of [6] cyclacene with those of coplanar anthracene molecule.

\begin{tabular}{|c|c|c|c|c|c|c|c|c|c|c|}
\hline Molecule & $\begin{array}{l}\text { C-H } \\
\text { sym. }\end{array}$ & $\begin{array}{c}\text { C-H } \\
\text { asym. }\end{array}$ & $\begin{array}{c}\text { C---Ca } \\
\text { sym. }\end{array}$ & $\begin{array}{l}\text { C---Ca } \\
\text { asym. }\end{array}$ & $\begin{array}{c}\text { C---Cc } \\
\text { sym. }\end{array}$ & $\begin{array}{l}\text { C---Cc } \\
\text { asym. }\end{array}$ & $\begin{array}{l}\delta \mathrm{CH} \\
\text { sym. }\end{array}$ & $\begin{array}{r}\delta \mathrm{CH} \\
\text { asym }\end{array}$ & $\begin{array}{l}\gamma \mathrm{CH} \\
\text { sym. }\end{array}$ & $\begin{array}{c}\gamma \mathrm{CH} \\
\text { asym. }\end{array}$ \\
\hline $\begin{array}{c}\text { [6] Cyclacene zig- } \\
\text { zag }\left(D_{6 h}\right)\end{array}$ & $\begin{array}{c}3067 \\
A_{1 g}\end{array}$ & $\begin{array}{c}3055 \\
\mathrm{~B}_{1 \mathrm{~g}}\end{array}$ & $\begin{array}{c}1531 \\
A_{1 g}\end{array}$ & $\begin{array}{c}1457 \\
\mathrm{~B}_{1 \mathrm{u}}\end{array}$ & ------- & $\begin{array}{c}1411 \\
\mathrm{~B}_{1 \mathrm{~g}}\end{array}$ & $\begin{array}{c}1222 \\
A_{1 g}\end{array}$ & $\begin{array}{c}1274 \\
B_{2 g}\end{array}$ & $\begin{array}{l}956 \\
A_{1 g}\end{array}$ & $\begin{array}{l}936 \\
E_{1 g}\end{array}$ \\
\hline $\begin{array}{c}\text { Anthracene } \\
\left(D_{2 h}\right)\end{array}$ & $\begin{array}{c}3156 \\
A_{g}\end{array}$ & $\begin{array}{c}3153 \\
B_{1 u}\end{array}$ & $\begin{array}{c}1592 \\
A_{g}\end{array}$ & $\begin{array}{c}1582 \\
B_{2 u}\end{array}$ & ------- & $\begin{array}{c}1671 \\
B_{3 g}\end{array}$ & $\begin{array}{c}1302 \mathrm{~A} \\
\mathrm{~g}\end{array}$ & $\begin{array}{c}1320 \\
B_{3 g}\end{array}$ & ----- & $\begin{array}{l}937 \\
B_{1 u}\end{array}$ \\
\hline
\end{tabular}

The result parallels that of Turker [26] who showed, on the basis of the Hückel treatment, that the electronic binding energy of the planar polyaromatic hydrocarbon are bigger in value than of the cyclacene molecule with similar number of benzene rings.
Finally, the calculations show that, similar to the carbon nanotubes [27-32], the charge densities are mainly concentrated at the circumferential carbon and hydrogen atoms of cyclacene. The axial carbon atoms have diminishing charges. And whereas the $\mathrm{H}$ atoms are positively charged, the $\mathrm{C}$ atoms are of the negative charge, Fig. 6.

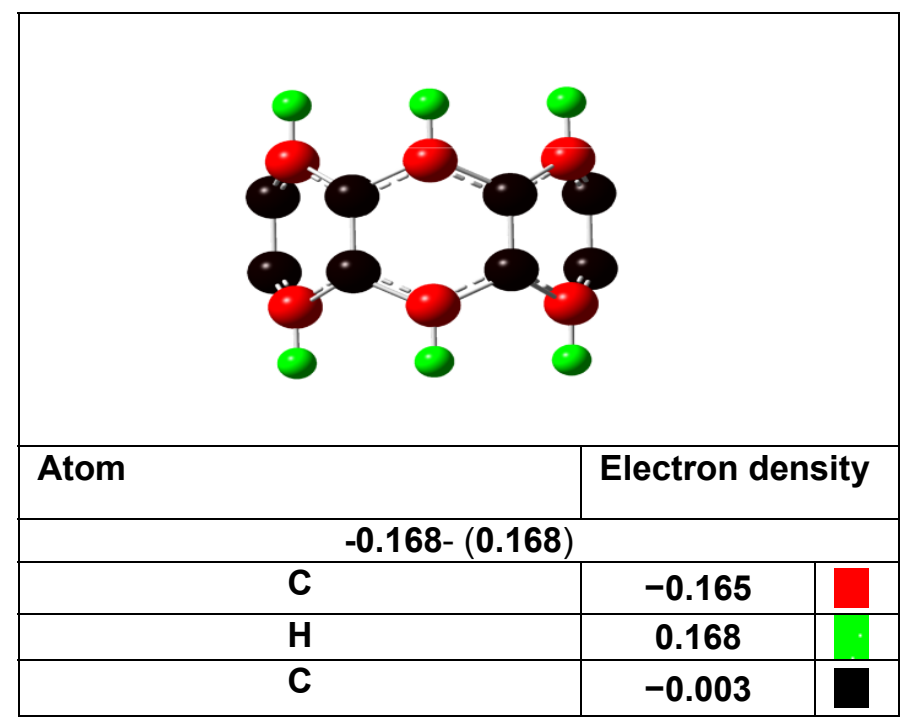

Fig. 6. Distribution of charge density at the atoms of [6] Cyclacene (zig-zag) molecule as calculated applying DFT (B3LYP/6-311G) method 


\section{REFERENCES}

M. Kaempgen, G. S. Duesberg and S. Roth, Appl. Surf. Sci. 252(2), 425-429 (2005).

I. Lukovitz, F. H. Karman, P. M. Nagy and E. Kalman, Croat. Chim. Acta, 80, 233-237 (2007).

T. Yao, Hao Yu, R. J. Vermelj and G. J. Bodwell, Phys. Rev.Lett. 80, 1-3, (2008).

M. S. Dresselhaus, G. Dresselhaus and P. Avouris, Carbon Nanotubes, Synthesis, Structures, Properties and Applications, Springer- Verlag, New York, 1996.

N. Anderson, A. Hartschuh, S. Cronin and L. Novotny, J. Am. Chem. Soc. 127, 2533-2537 (2005).

S. Ijima, Nature 354, 56-58 (1991); S. Iijima, T. Ichihashi, Nature 363, 603-605 (1993).

N. Hamada, S. Sawada, A. Oshiyama, Phys. Rev. Lett. 68,1579-1581(1992).

Y. Wang and X. Jing, Polym. Adv.Technol. 16, 344, (2005).

T. Durkop, S.A. Getty, E. Cobas and M.S. Fuhrer, Nano Lett.4,35-39, (2004).

B.I. Yacobson and R.E. Smalley, American Scientist, 85, 324-337, (1997).

R. Gleiter, B. Esser and S. Kornmeyer, Acc. Chem. Res. 16, 328-334 (1983).

M. Shanshal, Turk. J. Chem. 34, 269 - 275 (2010).

W. Kohn, and L. J. Sham, Phys. Rev., 140, A1133-A1138 (1965), P. Hohenberg, and W. Kohn, Phys. Rev., 136, B864-B871 (1964).

R. G. Parr and W. Yang, "Density-Functional Theory of Atoms and Molecules," Oxford, New York, p. 53. (1989.

G, See G03, User"s Manual, Gaussian, Inc. Pittsburgh, PA, 2003.

J. J. P. Stewart, J. Comp. Chem. 10, 209 (1989); J. J. P. Stewart, J. Comp. Chem. 10, 221, (1989); J. J. P. Stewart, J. Comp. Chem... 12, 320, (1991); M. J. S. Dewar, E. F. Healy, A. J. Holder, and Y. C. Yuan, J. Comp. Chem. 11, 541(1990).

Frisch, M. J.; Trucks, G. W.; Schlegel, H. B.; Scuseria, G. E.;Robb, M. A..; Cheeseman, J. R.; Montgomery, Jr., J. A.; Vreven,T.; Kudin, K. N.; Burant, J. C.; Millam, J. M.; lyengar, $\quad S$. $\quad$.; Tomasi, J.; Barone, V.; Mennucci, B.; Cossi, M.; Scalmani, G.; Rega, N.; Petersson, G. A.; Nakatsuji, H.; Hada, M.; Ehara, M.; Toyota, K.; Fukuda, R.; Hasegawa, J.; Ishida, M.; Nakajima, T.; Honda, Y.; Kitao, O.; Nakai, H.; Klene, M.; Li, X.; Knox, J. E.; P. Hratchian, H.; Cross, J. B.;

Adamo, C.; Jaramillo, J.; Gomperts, R.; Stratmann, R. E.; Yazyev, O.; Austin, A. J., Cammi, R.; Pomelli, C.; Ochterski, J. W.; Ayala, P. Y.; Morokuma, K.; Voth, G. A.; Salvador, P.; Dannenberg, J. J.; Zakrzewski, V. G.; Dapprich, S.; Daniels, A. D.; Strain, M. C.; Farkas, O.; Malick, D. K.; Rabuck, A. D.; Raghavachari, K.; Foresman, J. B.; Ortiz, J. V.; Cui, Q.; Baboul, A. G.; Clifford, S.; Cioslowski, J.; Stefanov, B. B.; Liu, G.;
Liashenko, A.; Piskorz, P.; Komaromi, I.; Martin, R. L.; Fox, D. J.; Keith, T.; Al-Laham, M. A.; Peng, C. Y.; Nanayakkara, A.; Challacombe, M.; Gill, P. M. W.; Johnson, B.; Chen, W.; Wong, M. W.; Gonzalez, C.; and Pople, J. A.; Gaussian, Inc. Pittsburgh, PA, 2003.

P. Bischoff, MOLEK9000 Program, OCI, Uni. Heidelerg, Private Communication.

L. Vitali, M. Burghard, M.A. Schneider, Y.Wu, Lei Liu, C. Jayanthi and K. Kem, Phys. Rev. Lett. 93, 136103, (2004).

R. Saito, G. Dresselhaus, M. S. Dresselhaus, Phys. Rev.B 61, 2981, (2000).

K.A. Ramani and H. Chadl, J. Phys. chem., B 110 (25), PP.12388-12393 (2006).

A.B. Dalton, J.N. Coleman, B. McCarthy, P.M. Ajayan, S. Lefrant, P. Bernier, W. Blau and H.J. Byme, J. Phys. Chem. B. 104, 10012, (2000).

U. Kuhlman, H. Jantoljak, N. Pfander, P. Bernier, C. Journet and C. Thomsen, Chem. Phys. Lett. 294, 234237, (1998).

A. Rochefort, P. Avouris, J. Phys. Chem. A 104, 98079811, (2000); L. Chico, L.X. Benedict, S.G. Louie, M.L. Cohen, Phys. Rev. B 54, 2600-2606 (1996); P.E. Pehrsson, W. Zhao, J. W. Baldwin, C. H. Song, J. Liu, S. Kooi and B. Zheng, J. Phys. Chem. B 107, 56905695 (2003).

G. Herzberg, "Molecular Spectra and Molecular Structure, Infrared and Raman Spectra of Polyatomic Molecules", van Nostrand Co. New York, 1971.

L. Turker, Turk. J. Chem. 24, 217-222 (2000).

S. Akita, Y. Nakayama, S. Mizooka, Y. Takano and Y. Okawa, "Nano Tweezers consisting of CNT", Appl. Phys. Lett. 79, pp. 1691-1693, (2001).

M. Krcmar, W.M. Saslow and A. Zangwill, J. Appl. phys. 93, pp. 3495-3500, (2003).

T. W. Odom, J. Huang, P. Kim and C. M. Lieber, J. Phy. Chem.104, 2794-2809, (2000).

S. Han and J. I. Hrm, Phys. Rev. B 61, 9986, (2000).

Li CY and Chou TW., Appl. Phys. Lett., 90, 174108, (2007).

H. N. Al-Ani, M.Sc. Thesis, College of Science, University of Baghdad, (2009). 